\title{
Three-Dimensional Visualization of Movement Qualities in Contemporary Dance
}

\author{
Rafael Kuffner dos Anjos \\ BlackBox - Universidade NOVA - \\ Faculdade de Ciências Sociais e \\ Humanas \\ Lisboa, Portugal \\ rkuffner@fcsh.unl.pt
}

\author{
Claudia Ribeiro \\ BlackBox - Universidade NOVA - \\ Faculdade de Ciências Sociais e \\ Humanas \\ Lisboa, Portugal \\ claudia.ribeiro@fcsh.unl.pt
}

\author{
Carla Fernandes \\ BlackBox - Universidade NOVA - \\ Faculdade de Ciências Sociais e \\ Humanas \\ Lisboa, Portugal \\ fcar@fcsh.unl.pt
}

\begin{abstract}
Analyzing and documenting contemporary dance movement data has proven to be a very difficult task due to the vast existence of styles and aesthetics which are characteristic of each individual choreographer. Indeed, identifying movement qualities with the intention of co-relating them to specific meanings and choreographic intentions is practically impossible without taking an artist-driven approach. Moreover, equipping dancers with wearable technology in order to accurately track each of their movements is only applicable in controlled laboratory scenarios, therefore not being a viable approach to analyze large scale real-world rehearsals or performances. This paper describes a visualization system that exposes movement qualities for dance through rendering effects. Combining optical flow analysis with depth video information, we were able to estimate the three-dimensional flow for each point in the cloud. This information was used to color point cloud videos with this information, showing potential to be used for the creation of different rendering effects, which are also discussed in this paper. The data used was captured in a non-intrusive manner during the creation process of a dance piece by Portuguese choreographer Rui Lopes Graça, enabling us to examine nuances and details of the rehearsals' process, which would not be replicable in a laboratory environment.
\end{abstract}

\section{CCS CONCEPTS}

- Applied computing $\longrightarrow$ Performing arts; $\bullet$ Computing methodologies $\rightarrow$ Point-based models;

\section{KEYWORDS}

Contemporary Dance, Motion Capture, Point Clouds, 3D Visualization, Optical Flow

\section{ACM Reference Format:}

Rafael Kuffner dos Anjos, Claudia Ribeiro, and Carla Fernandes. 2018. ThreeDimensional Visualization of Movement Qualities in Contemporary Dance. In MOCO: 5th International Conference on Movement and Computing, fune

Permission to make digital or hard copies of all or part of this work for personal or classroom use is granted without fee provided that copies are not made or distributed for profit or commercial advantage and that copies bear this notice and the full citation on the first page. Copyrights for components of this work owned by others than ACM must be honored. Abstracting with credit is permitted. To copy otherwise, or republish, to post on servers or to redistribute to lists, requires prior specific permission and/or a fee. Request permissions from permissions@acm.org.

MOCO, June 28-30, 2018, Genoa, Italy

(C) 2018 Association for Computing Machinery.

ACM ISBN 978-1-4503-6504-8/18/06 .. \$15.00

https://doi.org/10.1145/3212721.3212812
28-30, 2018, Genoa, Italy, Jennifer B. Sartor, Theo D'Hondt, and Wolfgang De Meuter (Eds.). ACM, New York, NY, USA, Article 4, 7 pages. https: //doi.org/10.1145/3212721.3212812

\section{INTRODUCTION}

Video has always been the preferred type of media when documenting a continuous real world event. Nowadays it has become more ubiquitous than ever, with a considerable amount of people carrying a camera with them all the time in their smartphone devices. Capturing and recording video data has transitioned from being a specialized task executed by experts, to being as commonplace as taking notes on a piece of paper.

In the context of contemporary dance, video is commonly used as the main documenting tool for choreographies, due to the high variety in styles, different steps, and aesthetic characteristics of distinct choreographers. Both dancers and choreographers commonly record different parts of a performance or a rehearsal not only for memorizing, but also for analyzing, evaluating, and comparing various executions of a dance segment. However, video information is often limited for both documentation and analysis. Two-dimensional video streams are a reduction of what can be seen in the real world, suffering from occlusions between different performers, and the fact that the sense of depth and space is distorted. Questions such as "how far apart must the dancer position his legs?" or "how close must both performers be from each other?" are difficult to answer from a single video. Contemporary dance is much more diverse than other codified dance types and is therefore much less easy to categorize. "Capturing contemporary dance practices requires a process of identifying repeatable sequences of movement that can be considered broadly representative of the genre." [34]. Moreover, precise analysis and evaluation of movement qualities and attempting to relate them to specific choreographic intentions is a difficult task with such a simple input.

Obtaining more detailed information typically requires either moving the dancers to a studio with specialized equipment (e.g. Vicon $^{1}$, Optitrack ${ }^{2}$ ), or equipping each individual dancer with wearables (e.g. Notch ${ }^{3}$ ). However, this is not feasible when one desires to capture stage performances, or even for small companies who are unable to afford the high cost of renting such facilities or gear for a recording session.

Recently, depth cameras and point cloud data have become more popular and commonplace through popular devices such as the

\footnotetext{
${ }^{1}$ https://www.vicon.com/

${ }^{2}$ http://optitrack.com/

${ }^{3}$ https://wearnotch.com/
} 
Microsoft Kinect. Also with the growing interest in Virtual and Augmented Reality, depth cameras are starting to be incorporated in consumer smartphones (e.g. Asus Zenfone AR, Iphone X). With this type of data being available, specialized visualization techniques can be developed to take advantage of this information and create informative applications for casual users.

We propose a visualization system based on depth videos which exposes movement qualities through different rendering effects and techniques. Our system only requires one or more depth cameras to capture the data, and can be set up in the rehearsal space, as we demonstrate with our data. We combine two-dimensional optical flow estimation with the depth differences in order to estimate a three-dimensional flow vector for each point in the cloud. This information was used to create two different visualizations (one for "intensity" and another for "directionality") discussed in this paper, which allows a choreographer to analyze dance-specific phenomena such as synchrony, marking, and intensity. We have tested the proposed system with real-world data captured from rehearsal sessions during the creation process of the piece "15 Dancers and uncertain time" by Portuguese choreographer Rui Lopes Graça, attesting the viability of the proposed approach. While previous work has addressed the limitations of $2 \mathrm{D}$ videos when it comes to documentation [25], presenting concrete movement information three-dimensionally has yet to be addressed.

This paper will start with a short state of the art review in movement qualities visualization, followed by a description of the developed system, and a short discussion of the obtained results.

\section{RELATED WORK}

Dance theorists focused on analyzing dance movement have developed analysis systems that provide tools to observe, analyse and describe movement patterns. Several researchers have proposed definitions of what should be considered a movement quality; in particular Blom and Chaplin [4] have put forward that it is "the distinctly observable attributes or characteristics produced by dynamics and made manifest in movement."

Dance theorist Rudolf Laban considered movement qualities to be a central notion in human motion. He developed the Laban Movement Analysis system, later extended by Ullman et al. [21], which provides a rigorous use of language to analyze functional and expressive movement. Laban Movement Analysis describes movement in terms of Body (What is moving?), Effort (How is it moving?), Space (Where is it moving?), and Shape (What relationship with the environment?) [20]. Laban Movement Analysis has been previously used in visualization of dance and planning choreography as described in [5, 7].

Current practices of dance researchers capturing movement qualities mostly rely on principles of field work [11], where "direct observation" of dance events in their natural setting was, and still is, the main method of dance studies. Within this practice, comprehensive technical recordings of dance, music, and texts, or of entire events, are realized through combining the following means: film/video [31], photography, sound recordings [32], and interviews with graphic notation [13], and further written documents such as questionnaires. Other approaches are rooted in anthropological research methods, and in this case the researcher takes a more participative and self-experienced role $[18,19]$.

In recent years developments in digital technology have provide tools, methods, and technologies for extracting and analyzing movement information from the human body [30]. These include sensors that allows determining fundamental properties of human movement, such as position, direction, velocity among others (e.g., video camera, Microsoft Kinect, Wiimote IR camera, Playstation move motion controller) [12]. Moreover, recently the use of biosensors such as BITalino has become more popular both in dance studies [23] and dance performances [3, 14, 22]. The advantage of using these kind of sensors is the ability to collect physiological data in real-time, such as muscle tension, EMG, and brain activity [8]. By contrast, on much higher-end settings, extremely recent equipment such as the HoloLens is being used in the EU-funded project WholoDance, which, although also drawing on the principles of Laban Movement Analysis [24, 27], have designed their own set of "Movement Principles" (Symmetry, Directionality, Balance, Alignment, Weight-bearing vs Gesturing, Gross vs. Fine Motorics, Coordination, Motion Through Space, Rhythm, Phrasing, and Stillness) which, according to the authors, serve as a "baseline of movement that is common to all dance forms" [34].

Having the ability to capture this kind of information has also contributed to the progress of the field of studies related to information visualization. Specifically in the area of dance studies, works such as $[9,15,25,28]$, have shown the possibilities of how movement can be visualized in radically new ways that allow to both analyze and document choreographic practices. Nevertheless, introducing a technological apparatus in a dance rehearsal or performance is not always the optimal solution, as it disturbs and also limits the movement of the dancers.

\section{SYSTEM DESCRIPTION}

Our system is composed of different modules responsible for data capture, post-processing, and visualization (see Fig. 1). This section will describe each aspect of our system, from possible capture setups, to each step of the processing pipeline that analyses the movement qualities and creates the three-dimensional videos.

\subsection{Data Capture}

As previously mentioned in Section 1, our goal is to capture the necessary data to analyze the movement qualities in a way that is as similar as possible to recording videos of a performance rehearsal. For that matter, we have chosen to use the Microsoft Kinect as a depth camera thanks to its portability. It can be connected to a laptop computer to record color and depth information as would be performed with a normal video camera. The implemented system just requires a pair of color and depth streams, and the latter can be obtained with any device or process.

We used three different setups for our study: single camera (Figure 2c), and a three camera wide-baseline setup with two different camera placements. During rehearsals, we used either a single camera setup, or three sensors positioned at three different corners of a right triangle (Figure $2 b$ ), outside the dancing area. For a more detailed capture in a specialized session, the three Kinect sensors were positioned in a triangular shape and the dancers were instructed 


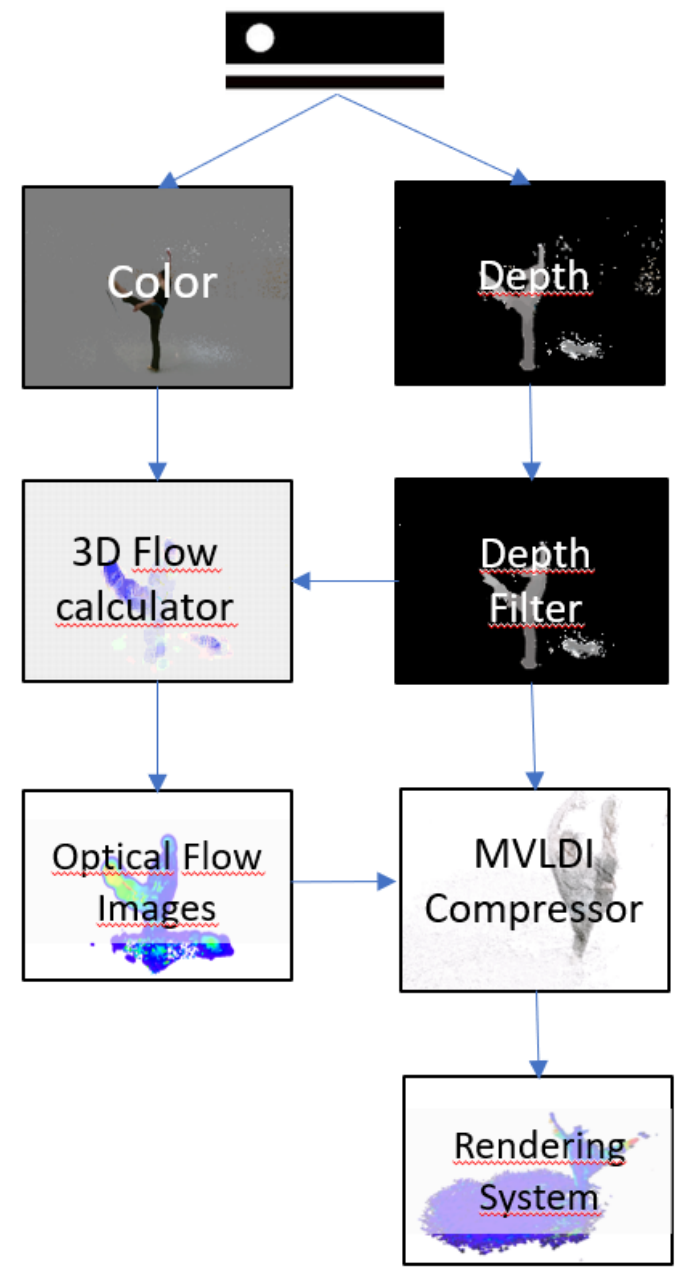

Figure 1: Flowchart of the data capture, processing, and visualization pipeline. Starting from depth maps and color images captured by a Kinect camera to a point cloud video with movement qualities

to perform the movement sequences at the center of this triangle (Figure 2a). For calibration of wide-baseline setups, we used the "Creepy Tracker" toolkit from Sousa et al. [29], which provides a calibration solution for different sensors in a local network in a short time.

\subsection{Data Processing}

The captured color and depth streams are used as input to the 3D Flow Calculator post-processing component. Due to the noisy nature of the depth data captured by the Kinect sensor, we preprocessed the depth data with a temporal and spatial median filter. This not only removes outliers which would indicate false motion in the dataset, but also minimizes the oscillation in the depth values that is characteristic of this type of sensor, which would also influence the results. We also applied a spatial median filter to the color stream, since the color pixels recorded are only the ones

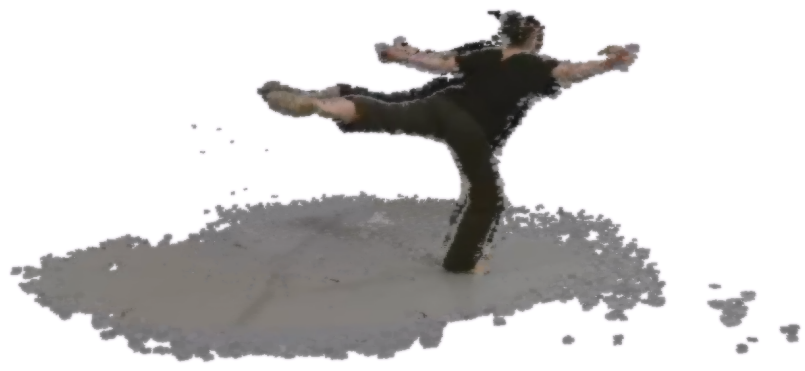

(a) 3 camera isolated setup

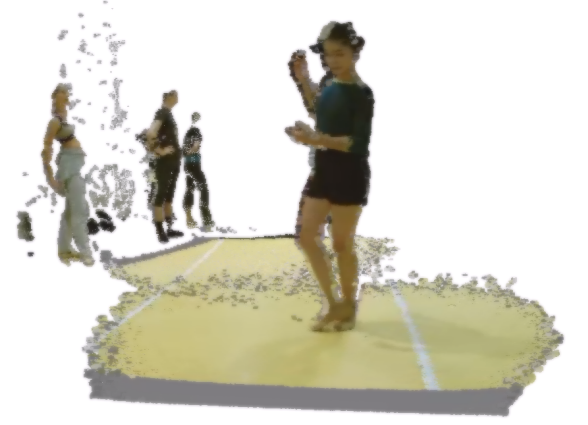

(b) 3 camera rehearsal setup

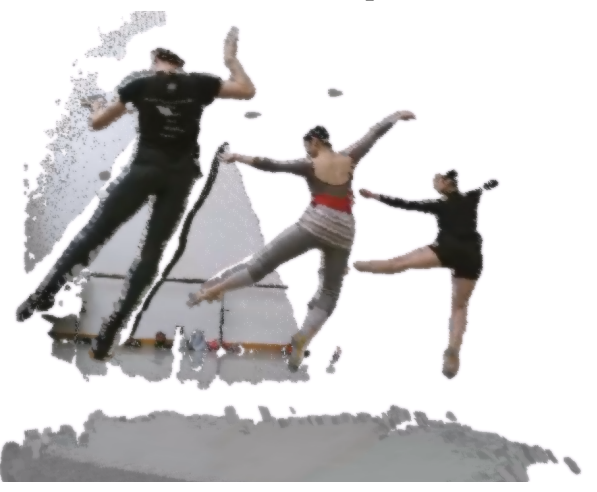

(c) Single camera setup

Figure 2: Example of three different point cloud videos from one and three camera setups. Using a wide-baseline setup avoids occlusions (seen in the background of $2 \mathrm{c}$

which directly represent a valid depth value, so it also contains several outliers which would heavily influence the flow estimation algorithm.

We use the Optical flow algorithm described by Farnebäck [10] to estimate the one frame motion estimation for each pixel in the color image. This two-dimensional vector is then combined with the depth displacements between two consecutive depth images, which is scaled according to a value $\epsilon$ so it has a similar magnitude to the flow output. The value $\epsilon$ is typically defined according to both 


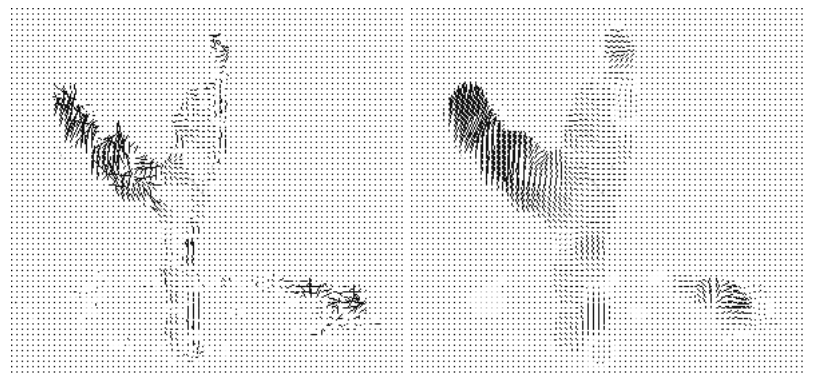

(a) Flow vectors with 4 pixel spacing
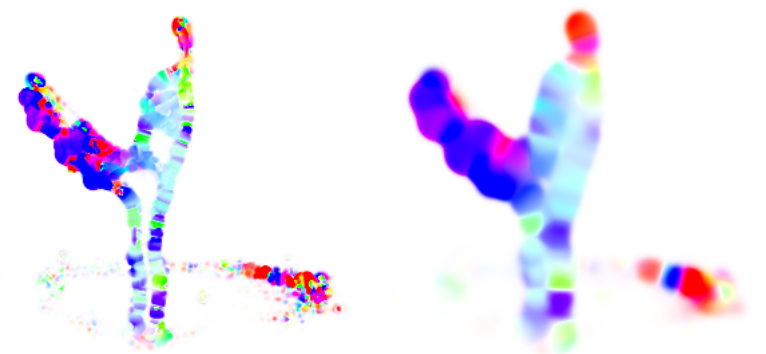

(b) Dense direction map, with each pixel representing its flow direction as a color.

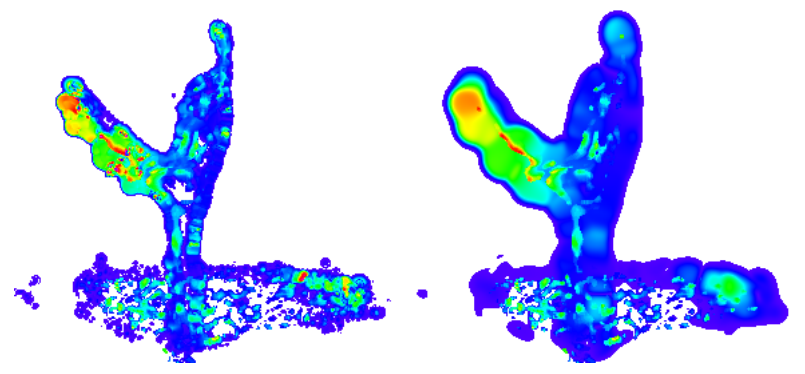

(c) Intensity estimation.

Figure 3: Information obtained after the data processing step. Left column used 5 pixel window size, right column had 30.

the type of scene being recorded and the proximity of the dancers in order to fine tune the obtained visual results. However, it can easily be estimated automatically using the camera calibration parameters, so that the image-space movement vectors can be transformed into world-space depth displacements, and vice-versa.

The parameters of the Farnebäck algorithm are chosen by the user according to the scenario at hand, with the most important being the window size, which defines the size of the algorithm's search window. Figure 3 shows the obtained output for different window sizes ( 5 for left column, 30 for right column). A bigger number will filter noisy results, but also blend localized movements in small areas (e.g. finger movements attributed to the whole forearm), while a too small number will lead to wrongly estimated directions due to lighting changes or noise in the input data.

The Farnebäck algorithm typically assigns the movement information to the pixels from where the object has moved, not to its destination. Thus, when writing the resulting color image files, we match it to the previous depth frame so that the colors are correctly applied to the rendered 3D points.

\subsection{Movement Qualities}

The three-dimensional flow vector $\vec{f}$ was used in our prototype to visualize two different aspects of the data: intensity and directionality. Different aspects of Rui Lopes Graça's work and the research being conducted about his compositional process could be observed through these visualizations (Section 5).

Figure 3 shows the the raw flow information, and the generated outputs. For directionality we represented the flow vector in polar coordinates, using $\rho$ ( 0 to 360 degrees) as the Hue value in the HSV color space.

The $r$ value which represents the intensity of the movement was mapped to the saturation, and the value component was kept as 1 at all times. We chose to map only the two-dimensional vector to the color space, instead of the three dimensions, since it allows a much easier interpretation and identification of synchrony between performers. Using the saturation and value parameters or an RGB color space has produced much unclearer results. Another factor considered in this decision was the fact that in wide-baseline setups the $\mathrm{Z}$ direction is represented as a different axis, so it is still considered in the visualization if one desires to observe synchrony along that axis.

For the intensity visualization, we used $\|\vec{f}\|$ mapped to the HSV space, varying the Hue value from 260 (blue) at the lowest intensity to 0 (red) at the maximum intensity in order to create a visualization similar to a heat map, which is a known concept by most users (e.g. weather reports, traffic information). The saturation component was set to 1 whenever $\|\vec{f}\|$ different than 0 , and the value was set as 1 throughout the whole image.

\subsection{Visualization}

The resulting direction or intensity images are saved to disk, replacing the originally captured color images in the compression process. We use the Multiview Layered Depth Image (MVLDI) by Anjos et al. [1], which compactly encodes single frames of three-dimensional videos of varied baselines. Color information was compressed using the H.264 algorithm [26], which is implemented in the NVIDIA video codec SDK (NVEnc) ${ }^{4}$. In order to create a better splat-based visualization of our data, we also compressed the estimated normal vectors from the point cloud into a separate color stream. We directly map the $X Y Z$ coordinates in the $R G B$ color space, and use the $a$ value to keep a bit mask of the signal of each coordinate:

$$
\begin{array}{ll|l|l|l|l|l|l|l|l|}
\hline a & 0 & 0 & 0 & 0 & 0 & \operatorname{sig}_{x} & \operatorname{sig}_{y} & \operatorname{sig}_{z} \\
\hline
\end{array}
$$

The depth stream was encoded using the RVL algorithm proposed by [33], a combination of run length encoding and variable length encoding compressing each frame independently using the process described in the paper.

Rendering was implemented in C++ using OpenGL and GLSL. The normal and color streams are decoded normally as video streams (FFmpeg and NVENC were used in our case), updating pixel buffers at $30 \mathrm{fps}$, which will be available in texture memory by the vertex

\footnotetext{
${ }^{4}$ https://developer.nvidia.com/nvidia-video-codec-sdk, accessed october 2017
} 
shader. Depth data is decoded using the RVL algorithm [33] and updated into an array buffer which is aligned with UV coordinates in a vertex array passed onto the vertex shader.

The vertex shader transforms the $(u, v, d)$ image-space coordinates into $(x, y, z)$ world coordinates using the depth camera intrinsics matrix. Then, each point is multiplied by the extrinsics matrix representing the calibrated position of that particular stream (contained in the MVLDI output).

\section{RESULTS}

\subsection{Visualization of Movement Qualities}

Data was collected over the course of several days of rehearsal, using different setups. We captured individual data from five different performers using the wide-baseline setup, two different group rehearsals with also a wide-baseline setup, and six different instances from group rehearsals with one single camera. The length of the datasets varied between 30 seconds to two minutes, and no special instructions were given to the performers, except not to touch the cameras and to keep the performance inside the captured space in the isolated setup.

All the datasets were processed and converted to 3D videos and can be visualized interactively with free choice of viewpoint by the users. The complete set of results can be seen in the video attached to this publication. This data can also be easily displayed through virtual and augmented reality headsets, for a more immersive experience.

Figure 4 shows six different snapshots of the different results obtained with the presented approach. The top row (Figures 4 a to 4c) shows the obtained point cloud visualizations for movement intensity, highlighting different aspects that can be observed through this type of output. Figure 4a shows an example of a dancer quickly going through movements before a group rehearsal (other dancers waiting in the background), hence the low intensity in her movements in comparison with a full out performance in Figure 4c. The same phenomena can be seen in Figure $4 \mathrm{~b}$ where the leftmost dancer has a lower intensity than the other performers, due to the fact that he is not dancing with full intensity, but just "marking" his steps.

The bottom row shows the direction visualization in group rehearsals. This visualization was particularly effective in situations with more than one dancer where relations can be created. Figure $4 \mathrm{~d}$ shows a straightforward synchronized entrance on stage by all dancers. A similar situation can be seen in Figure 4e, but having two different groups of dancers moving in opposing directions. A case where two dancers have a slight lack of synchrony between both of their performances can be seen in Figure 4f, where although having the same position and being seemingly synchronized, their legs are moving in opposing directions during the highlighted step.

\subsection{Evaluation}

We met with choreographer Rui Graça to have an expert opinion on the obtained results, and possible use-case scenarios of this tool. The process and the meaning of the color maps was explained, and open-ended questions concerning the tool, its visual results, and his personal work were posed.
Two main topics dealt with the applicability of this system to his own daily workflow, and to the usefulness of this system when observing and researching dance in general.

Rui Graça replied that watching recordings of his work and rehearsals is not his usual modus operandi and would not fit his particular day-to-day workflow. Nonetheless, he indicated that he was aware of other choreographers who usually recorded videos to reproduce choreographies from previous days or to manage the evolution of a piece. In this specific creation, several sessions had been video recorded to help recall previous work, possibly due to a tighter schedule and a higher number of dancers. Recording typically was a task carried out by the choreographer's assistants, who would use their mobile phones to capture videos during rehearsals, re-watch them, and show them to him or to the dancers for clarification. This workflow is typical in the work of most choreographers especially in academic scenarios [17], and where filming and watching rehearsals is a fundamental part of everyday sessions.

As a medium for analysis of other choreographers' works, he agreed with the usefulness of the tool. He stated that the tool can be used to study his or other artists' works. This practice is specifically relevant in the academic community [16] for the study and analysis of different movements of dance, where annotated data is widely used. Regarding the color mapping, Rui Lopes Graça used it without prompt, casually identifying marking and synchrony while watching the rendered data, thereby confirming that specific usefulness and usability of the system. He considered the produced visualizations as a strong inspiration for future works, as well as a piece of art in and of itself.

To follow up on the topic of inspiration for future work, he indicated that he participates live to other staged performances, only resorting to its recording when not able to attend in person. We believe that the three-dimensional aspect of the visualizations will help bridge the gap between video recordings and reality when integrated with VR and AR systems.

\section{DISCUSSION}

Extracting and analyzing movement qualities that can characterize the aesthetic qualities of a choreographer or between different styles of dance is a central and active research in Dance Studies. With this work our goal is not to define which movement qualities are most relevant in dance but to propose a computational tool that could potentially help inform the frameworks developed by projects such as the WholoDance [6] or Bevilacqua et al. Gesture Follower [2]. Specifically, by using this tool it is possible to objectively measure direction and movement intensity for each body part. An example of applications could be for comparing the physical intensities between classical ballet and contemporary dance [35]. Also, analyzing side-by-side performances from different choreographers may help differentiate how they use direction and intensity in their respective compositional processes. A common practice in dance rehearsals is to analyze the video recordings at the end of the sessions. The ability to be able to observe raw data side by side with post-processed data could also serve as a tool for both dancers and choreographers alike to observe movements which need to be improved or what certain dancers need to change to be synchronous with the group. 


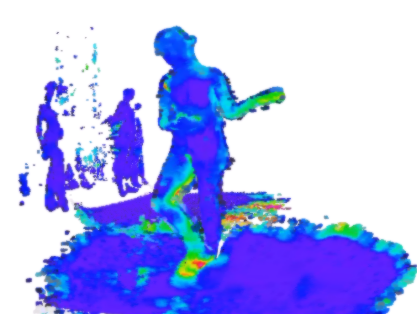

(a) Marking

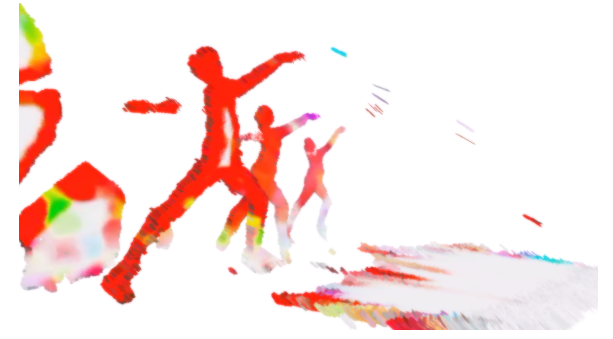

(d) Synchronized movement

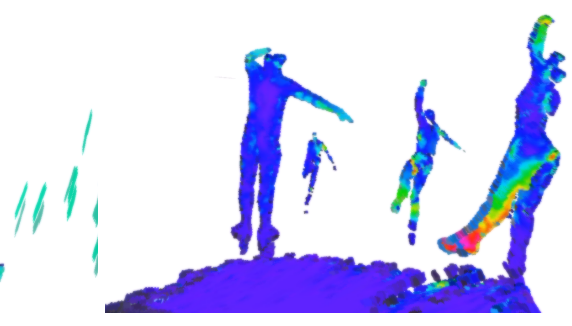

(b) Difference in intensities

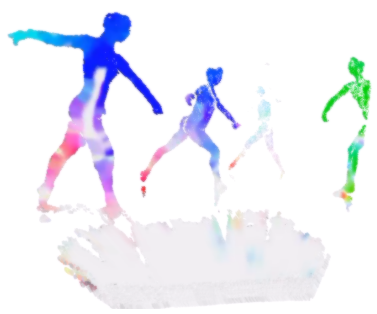

(e) Opposing directions

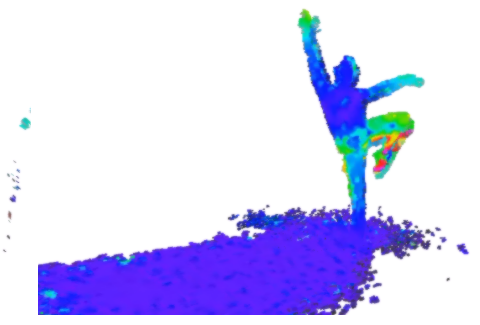

(c) Full out dance

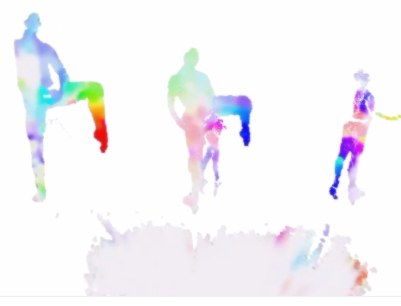

(f) Lack of synchrony

Figure 4: Six snapshots of the obtained results. Top row shows intensity visualization, bottom row the movement direction.

Movement intensity data can also be used to compare a dancer's performance throughout the rehearsal process, specifically compare which movements are physically more demanding for each dancer. Direction data can be used to analyze aspects related to synchrony between groups of dancers.

The information obtained after the data processing step (directionality and intensity) can be used for quantitative analyses, allowing other types of applications to be developed. Longer recordings can be segmented by intensity for quicker lookup of specific moments. Statistics and condensed visualizations can also be obtained from these intermediate results allowing one to look at a recording session as a whole.

We have used this tool to analyze the creative process of Portuguese choreographer Rui Lopes Graça. The piece we accompanied for our research, Quinze Bailarinos e Tempo Incerto, was composed under the artistic Direction of Joõo Penalva, who gave it a rather geometrical and non-narrative orientation. Together, its authors have devised a set of compositional rules, which started with attributing to each dancer a different code of four digits to be memorized and used as a prompt to start creating movement material. The idea was that they should be concentrated on abstract concepts, such as numbers and would "draw" their numbers in space by moving their necks, shoulders, torsos, and hips. Out of these initial movements, they then improvised several other sequences until the shape of the numbers in their bodies was not recognizable anymore. This compositional choice has motivated by our attempt to create visualizations of a space-bound parameter such as the "directionality" of the movements and of a qualitative parameter such as the "intensity" of the executed movements. These aspects reflect only some of the aspects we have been researching around the work of Rui Graça, but they complement our global research on this dance piece.

\section{CONCLUSIONS}

This paper presents a complete movement-qualities visualization system for dance, based on 3D videos. Using depth and color images, we are able to estimate a three-dimensional flow for each captured point of the performers, and generate clear visual representations in an interactive visualization system based on point clouds. Moreover, our system is less intrusive than similar movement capture solutions which typically require a higher investment in wearable gear, expensive devices, or dislocating the troupe of dancers to a specialized motion capture laboratory.

Real world data was captured and utilized in all steps of the process, proving the usability of this tool. The proposed solution can be compared to filming a rehearsal with different cameras, and processing the data for a richer visualization. Usually, this requires one or more Kinect sensors connected to a computer in order to capture the data, but with depth cameras currently being incorporated in mobile phones, capturing three-dimensional data will soon become a common activity.

The presented system can be used as a powerful tool for video analysis for both choreographers and dancers in reviewing their performances and rehearsals, comparing movements between different dancers, or the same dancer in different moments in time. Movement intensity and directionality were used to more clearly detect phenomena such as marking, synchrony, changes in direction, performance intensity, and other key aspects of a specific choreographer's work.

Our work adds a quantitative layer to the analysis of movement qualities, allowing scholars to more accurately analyze performances in a non-intrusive way. Going forward, our purpose is to use the generated information to create richer and clearer visualizations, describing more specifically each one of the studied phenomena. Also, numerical analysis and statistics can be incorporated in a 3D video player application in order not only to summarize sections of 
the produced video, but also to quickly allow looking up for specific moments in the performance. Combining this technology with human tracking software can allow us to precisely estimate intensity and movement in certain body parts, which can also be a powerful tool for the field of Gesture studies and non-verbal communication.

\section{ACKNOWLEDGEMENTS}

This work was supported by the European Research Council under the BlackBox project (Grant Agreement Ref. 336200). We would also like to thank the Portuguese National Ballet Company (CNB), Rui Lopes Graça, João Penalva, and all the dancers that have accepted to be video recorded during this project.

\section{REFERENCES}

[1] Rafael dos Anjos, João Madeiras Pereira, José António Gaspar, and Carla Fernandes. 2017. Multiview layered depth image. Journal of WSCG 25(2) (2017), $115-122$.

[2] Frédéric Bevilacqua, Bruno Zamborlin, Anthony Sypniewski, Norbert Schnell, Fabrice Guédy, and Nicolas Rasamimanana. 2009. Continuous realtime gesture following and recognition. In International gesture workshop. Springer, 73-84.

[3] Johannes Birringer. 2004. Interactive dance, the body and the Internet. Fournal of Visual Art Practice 3, 3 (2004), 165-178.

[4] Lynne Anne Blom and L Tarin Chaplin. 1982. The intimate act of choreography. University of Pittsburgh Pre.

[5] Tom Calvert, W Wilke, Rhonda Ryman, and Ilene Fox. 2005. Applications of computers to dance. IEEE computer Graphics and Applications 25, 2 (2005), 6-12.

[6] Antonio Camurri, Corrado Canepa, Nicola Ferrari, Maurizio Mancini, Radoslaw Niewiadomski, Stefano Piana, Gualtiero Volpe, Jean-Marc Matos, Pablo Palacio, and Muriel Romero. 2016. A system to support the learning of movement qualities in dance: a case study on dynamic symmetry. In Proceedings of the 2016 ACM International Foint Conference on Pervasive and Ubiquitous Computing: Adjunct ACM, 973-976.

[7] Kristin Carlson, Thecla Schiphorst, and C Shaw. 2011. ActionPlot: a visualization tool for contemporary dance analysis. In Proceedings of the International Symposium on Computational Aesthetics in Graphics, Visualization, and Imaging. ACM, 113-120.

[8] Hugo Plácido da Silva, Ana Fred, and Raúl Martins. 2014. Biosignals for everyone IEEE Pervasive Computing 13, 4 (2014), 64-71.

[9] Scott DeLahunta and Frédéric Bevilacqua. 2007. Sharing descriptions of movement. International fournal of Performance Arts and Digital Media 3, 1 (2007), $3-16$.

[10] Gunnar Farnebäck. 2003. Two-frame motion estimation based on polynomial expansion. In Scandinavian conference on Image analysis. Springer, 363-370.

[11] Anca Giurchescu and Lisbet Torp. 1991. Theory and methods in dance research a European approach to the holistic study of dance. Yearbook for Traditional Music 23 (1991), 1-10.

[12] Forouzan Golshani, Pegge Vissicaro, and Youngchoon Park. 2004. A multimedia information repository for cross cultural dance studies. Multimedia Tools and Applications 24, 2 (2004), 89-103.

[13] Don Herbison-Evans. 1988. Dance, video, notation and computers. Leonardo (1988), 45-50.

[14] Aurie Y. Hsu and Steven T. Kemper. 2010. Shadows No. 4: Belly Dance and Interactive Electroacoustic Musical Performance. In CHI '10 Extended Abstracts on Human Factors in Computing Systems (CHI EA '10). ACM, New York, NY, USA, 3117-3120. https://doi.org/10.1145/1753846.1753929

[15] Sally Jane Norman. 2006. Generic versus idiosyncratic expression in live performance using digital tools. Performance Research 11, 4 (2006), 23-29.

[16] Stephan Jürgens. 2016. Three methods of designing a workflow with multimodal video annotation in interdisciplinary choreographic processes.. In Multimodal ity and Performance, Carla Fernandes (Ed.). Newcastle upon Tyne: Cambridge Scholars Publishing, 159-178.

[17] Stephan Jürgens. 2017. How to communicate on the verge of collapse. Choreo graphic Practices 8, 1 (2017), 89-109. https://doi.org/doi:10.1386/chor.8.1.89

[18] Adrienne Lois Kaeppler. 1978. Dance in anthropological perspective. Annual Review of Anthropology 7, 1 (1978), 31-49.

[19] Adrienne L Kaeppler. 2000. Dance ethnology and the anthropology of dance. Dance Research fournal 32, 1 (2000), 116-125.

[20] Rudolf Laban. 1994. La maîtrise du mouvement. Arles, Actes Sud (1994), 48

[21] Rudolf Laban and Lisa Ullmann. 1971. The Mastery of Movement. (1971).

[22] Performance Art Magazine. 2002. Dance and media technologies. PAF: A fournal of Performance and Art 24, 1 (2002), 84-93.
[23] Teoma J Naccarato and John Maccallum. 2017. Critical Appropriations of Biosensors in Artistic Practice. In Proceedings of the 4th International Conference on Movement Computing. ACM, 3.

[24] Valerie Preston-Dunlop. 1983. Choreutic concepts and practice. Dance Research 1, 1 (1983), 77-88.

[25] Claudia Ribeiro, Rafael Kuffner dos Anjos, and Carla Fernandes. 2017. Capturing and Documenting Creative Processes in Contemporary Dance. In Proceedings of the 4th International Conference on Movement Computing. ACM, 7.

[26] Iain E Richardson. 2004. H. 264 and MPEG-4 video compression: video coding for next-generation multimedia. John Wiley \& Sons.

[27] Sarah Rubidge, V Preston-Dunlop, and A Sanchez-Colberg. 2002. Dance and the Performative: A Choreological Perspective, Laban and Beyond. (2002).

[28] Gongbing Shan, Peter Visentin, and Tanya Harnett. 2010. A novel use of 3D motion capture: Creating conceptual links between technology and representation of human gesture in the visual arts. Leonardo 43, 1 (2010), 34-42.

[29] Maurício Sousa, Daniel Mendes, Rafael Kuffner Dos Anjos, Daniel Medeiros, Alfredo Ferreira, Alberto Raposo, João Madeiras Pereira, and Joaquim Jorge. 2017. Creepy Tracker Toolkit for Context-aware Interfaces. In Proceedings of the 2017 ACM International Conference on Interactive Surfaces and Spaces (ISS '17). ACM, New York, NY, USA, 191-200. https://doi.org/10.1145/3132272.3134113

[30] Pattarawut Subyen. 2014. Mapping, Meaning, and Motion: An Artistic Framework for Visualizing Movement Quality. Ph.D. Dissertation. Communication, Art \& Technology:

[31] Emily Twitchett, Manuela Angioi, Yiannis Koutedakis, and Matthew Wyon. 2009. Video analysis of classical ballet performance. Fournal of Dance Medicine \& Science 13, 4 (2009), 124-128.

[32] Tamas Ungvary, Simon Waters, and Peter Rajka. 1992. NUNTIUS: A computer system for the interactive composition and analysis of music and dance. Leonardo (1992), 59-68.

[33] Andrew D. Wilson. 2017. Fast Lossless Depth Image Compression. In Proceedings of the 2017 ACM International Conference on Interactive Surfaces and Spaces (ISS '17). ACM, New York, NY, USA, 100-105. https://doi.org/10.1145/3132272.3134144

[34] Karen Wood, Rosemary E Cisneros, and Sarah Whatley. 2017. Motion Capturing Emotions. Open Cultural Studies 1, 1 (2017), 504-513.

[35] Matthew A Wyon, E Twitchett, M Angioi, F Clarke, G Metsios, and Yiannis Koutedakis. 2011. Time motion and video analysis of classical ballet and contemporary dance performance. International journal of sports medicine 32, 11 (2011), 851-855. 